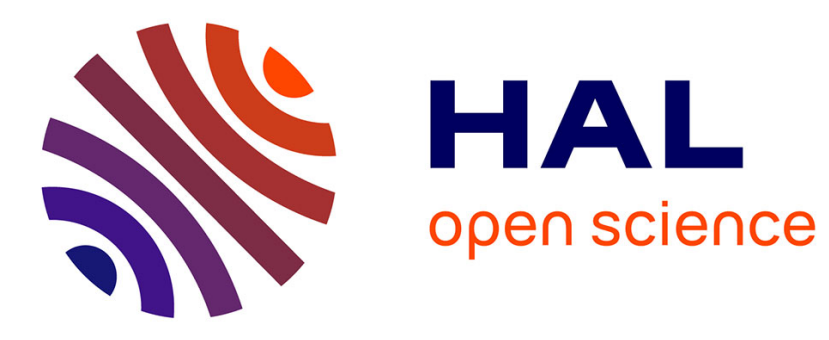

\title{
Non-destructive evaluation of 3D microstructure evolution in strontium titanate
}

\author{
Andreas Trenkle, Melanie Syha, Wolfgang Rheinheimer, Patrick G. Callahan, \\ Lily Nhuyen, Wolfgang Ludwig, William C. Lenthe, Mclean Echlin, Tresa \\ Pollock, Daniel Weygand, et al.
}

\section{To cite this version:}

Andreas Trenkle, Melanie Syha, Wolfgang Rheinheimer, Patrick G. Callahan, Lily Nhuyen, et al.. Non-destructive evaluation of 3D microstructure evolution in strontium titanate. Journal of Applied Crystallography, 2020, 53, pp.349-359. 10.1107/S160057672000093X . hal-02463919

\section{HAL Id: hal-02463919 https://hal.science/hal-02463919}

Submitted on 9 Dec 2020

HAL is a multi-disciplinary open access archive for the deposit and dissemination of scientific research documents, whether they are published or not. The documents may come from teaching and research institutions in France or abroad, or from public or private research centers.
L'archive ouverte pluridisciplinaire HAL, est destinée au dépôt et à la diffusion de documents scientifiques de niveau recherche, publiés ou non, émanant des établissements d'enseignement et de recherche français ou étrangers, des laboratoires publics ou privés. 


\title{
Non-destructive evaluation of $3 \mathrm{D}$ microstructure evolution in strontium titanate
}

\author{
A. Trenkle ${ }^{a}$ M. Syha, ${ }^{a}$ W. Rheinheimer,${ }^{a, b}$ P.G. Callahan, ${ }^{c}$ L. NGuyen, ${ }^{d}$ \\ W. Ludwig,${ }^{e}$ W. Lenthe, ${ }^{d, f}$ M.P. Echlin,${ }^{f *}$ T.M. Pollock,${ }^{f}$ D. Weygand,${ }^{a}$ \\ M. De Graef ${ }^{d}$ M.J. Hoffmann ${ }^{a}$ And P. Gumbsch ${ }^{a, g}$ \\ ${ }^{a}$ Institute for Applied Materials IAM, Karlsruhe Institute of Technology KIT, Straße \\ am Forum 7, 76131 Karlsruhe, Germany, ${ }^{b}$ Materials Engineering, Purdue \\ University, 707 West Stadium Ave, West Lafayette, IN 47907, USA, ${ }^{\circ}$ Materials \\ Science and Technology Division, The US Naval Research Laboratory, Washington, \\ D.C. 20375, USA, ${ }^{d}$ Department of Materials Science and Engineering, Carnegie \\ Mellon University, 5000 Forbes Avenue, Pittsburgh PA 15213, USA, éEuropean \\ Synchrotron Radiation Facility ESRF, 6 rue Jules Horowitz, Grenoble 38043, \\ France, ${ }^{f}$ Materials Department, University of California - Santa Barbara, Santa \\ Barbara, CA 93106-5050, USA, and ${ }^{g}$ Fraunhofer IWM, Wöhlerstr. 11, 79108 \\ Freiburg, Germany. E-mail: mechlin@ucsb.edu
}

\begin{abstract}
Non-destructive X-Ray diffraction contrast tomography (DCT) imaging was used to characterize the microstructure evolution in a polycrystalline bulk strontium titanate specimen. Simultaneous acquisition of diffraction and absorption information allows for the reconstruction of shape and orientation of more than 800 grains in the specimen
\end{abstract}

PREPRINT: Journal of Applied Crystallography A Journal of the International Union of Crystallography 
as well as porosity. Three-dimensional (3D) microstructure reconstructions of two coarsening states of the same specimen are presented alongside a detailed exploration of the crystallographic, topological and morphological characteristics of the evolving microstructure. The overall analysis of the 3D structure shows a clear signature of the grain boundary anisotropy, which can be correlated to surface energy anisotropy: the grain boundary orientation distribution function shows an excess of $<100>$ oriented interfaces with respect to a random structure. The results are discussed in the context of interface property anisotropy effects.

\section{Introduction}

Microstructural evolution of polycrystalline materials has been studied intensively by modeling and experiments (Burke \& Turnbull, 1952; Hillert, 1965; Holm \& Bataille, 2012). While there is a general agreement that microstructure characteristics, e.g. crystallographic orientation, interface orientation, grain morphology and grain size, influence the macroscopic material properties (Hall, 1954; Avrami, 1941), little is known on the relationship between the anisotropy of the interface properties, growth kinetics and resulting changes in the morphology of the grains within a polycrystal. This is because it is very difficult to infer such information on the microstructure evolution indirectly via (mostly 2D) statistical measures. On the other hand, it was hitherto impossible to acquire precise experimental information about the polycrystalline 3D microstructure of the same bulk specimen by non-destructive methods which would directly give access to information on the microstructure evolution. This has become possible with the development of the X-ray microscopy and diffraction contrast tomography (DCT) methods (Ludwig et al., 2008; Ludwig et al., 2009; Zhang et al., 2018). DCT is employed here to study grain growth in a sample of strontium titanate before and after a heat treatment step.

IUCr macros version 2.1.6: 2014/01/16 
Strontium titanate is known to be a highly anisotropic material in terms of deformation characteristics (Gumbsch et al., 2001), interface energy and mobility (Sano et al., 2003; Lee et al., 2003; Chung \& Kang, 2003; Kang et al., 2003; Rheinheimer et al., 2015b; Rheinheimer et al., 2015a). Investigations on the grain growth kinetics of strontium titanate show a very unusual deviation from the expected Arrhenius type behavior by displaying a dramatic decrease in the grain growth rate by three orders of magnitude when increasing the annealing temperature above a critical temperature (Bäurer et al., 2009b; Amaral et al., 2013; Rheinheimer \& Hoffmann, 2015). The mechanistic origin of this anomaly is not clear so far, but it is apparent that certain grain boundaries must abruptly change from a fast-moving low-temperature type to a slow-moving high-temperature type (Rheinheimer \& Hoffmann, 2015; Rheinheimer et al., 2019). A change in grain boundary faceting of strontium titanate with temperature was proposed to explain the non-Arrhenius behavior (Bäurer et al., 2010).

Rheinheimer et al. (Rheinheimer et al., 2015a) measured the temperature dependent distribution of the grain boundary planes occurring in bulk strontium titanate specimens and found an increase in the frequency of low energy $\{100\}$ grain boundary planes. However, the methods used in (Rheinheimer et al., 2015a) are based on a statistical analysis of 2D microstructure data. A direct observation of specific boundaries or changes due to grain growth is not possible with the used methods.

After the first successful applications of X-ray diffraction contrast tomography to strontium titanate (Syha et al., 2012; Syha et al., 2013; Lenthe et al., 2015), the present report focuses on the three-dimensional characterization of the evolution of the overall microstructure based on three-dimensional volume information at a local scale during an annealing experiment. Such full 3D reconstruction before and after microstructural changes is expected to provide grain-by-grain information on the structural evolution, a kind of information that has previously not been available.

IUCr macros version 2.1.6: 2014/01/16 


\section{Methods and Materials}

The bulk material for the tomography specimen was prepared from strontium titanate $\left(\mathrm{SrTiO}_{3}\right)$ powders processed by the mixed oxide/carbonate route from strontium carbonate $\left(\mathrm{SrCO}_{3}\right)$ and titania $\left(\mathrm{TiO}_{2}\right)$ (both $99.9+\%$, Sigma Aldrich Chemie, Taufkirchen, Germany) using a molar Sr/Ti ratio of 0.996. The material was sintered for $1 \mathrm{~h}$ at $1600^{\circ} \mathrm{C}$ in oxygen, cut and ground to the cylindrical shape $(\sim 300 \mu \mathrm{m}$ diameter $)$ required for tomography investigations. Detailed information on the fabrication process of the tomography specimens and microstructure investigations of the material are provided in (Bäurer et al., 2009b; Bäurer et al., 2009a; Syha et al., 2012).

DCT measurements were performed on the specimen using the setup and technical details as described in (Ludwig et al., 2009; Reischig et al., 2013). The specimen was annealed ex-situ for $1 \mathrm{~h}$ at $1600^{\circ} \mathrm{C}$ in air between two DCT scans. The DCT scans were performed at the materials science beamline ID11 of the European Synchrotron Radiation Facility, with a beam energy of $36 \mathrm{keV}$ at an effective resolution of $1.4 \mu \mathrm{m}$ before the anneal and $0.7 \mu \mathrm{m}$ thereafter. Data was acquired in full $360^{\circ}$ scans, taking in total 7200 images with an angular stepping of $0.05^{\circ}$. Data analysis was performed based on Friedel pairs as described in (Ludwig et al., 2009; Syha et al., 2012). After assembly of the grain volumes into the common sample volume, the resulting grain map will contain small regions which remain unassigned (Ludwig et al., 2009). A space filling microstructure is then obtained by a uniform dilation step (Syha et al., 2013). The resulting microstructure reconstructions are given as voxel data with crystallographic orientation for each grain. By comparison to electron back-scatter diffraction (EBSD) cross-sections of the annealed state of the specimen the spatial resolution at the grain boundaries was estimated better than $2 \mu \mathrm{m}$ (Syha et al., 2013). The grains are given a unique labeling in the initial structure roughly corresponding to their size, with the largest grain labeled \#1.

IUCr macros version 2.1.6: 2014/01/16 
For the initial state of the specimen, the DCT scans were complemented by absorption contrast tomography reconstructions yielding shape and location of residual pores inside the specimen. Post-annealing, a separate phase contrast tomography (PCT) dataset (Cloetens et al., 1997) was acquired using the same setup, but with a larger sample detector distance. The resulting edge enhancement due to free space propagation (Fresnel diffraction) increases the visibility of small pores and provides shape and distribution of pores inside the specimen with a resolution well below $1 \mu \mathrm{m}$ (resulting pixel size $0.56 \mu \mathrm{m})$. The two reconstructions were mapped to the smaller voxel size and their reference frames were first aligned using volume registration. Then an algorithm for automatic alignment and grain identification between the 3D datasets, presented in (Lenthe et al., 2015) and integrated with the DREAM.3D software suite (Groeber \& Jackson, 2014), is used on the present data. This algorithm identifies the same grains in two datasets by their centroid position in the 3D structure, grain orientation, overlapping grain volumes, and common neighbor analysis (Lenthe et al., 2015). This algorithm results in a voxel-scaled dataset alignment, which accounts for any magnification discrepancies, and results in a well aligned exterior sample surface.

Surface grains, ones that impinge on the sample exterior, have been removed from the analysis in order to focus on grains with all sides adjacent to other grains. This biased grain removal technique was necessary in order to provide as many grains as possible for analysis, however with larger 3D volumes one could implement unbiased techniques (Russ \& Dehoff, 2000).

\section{Results}

\subsection{D Microstructure}

Three-dimensional microstructure reconstructions of the specimen in both annealing states are presented in Figure 1(a) and (b). The overall shape is almost identical.

IUCr macros version 2.1.6: 2014/01/16 
Individual surface grains in both annealing states can easily be identified by their color, which is assigned to crystallographic orientation of the grain. The second scan was performed on a slightly smaller subvolume of the specimen. Therefore, the initial specimen state dataset was reduced to a similar subvolume, as shown in Figure 1. The alignment procedure (Lenthe et al., 2015) described in the Methods Section 2 results in a dataset alignment that is resilient to magnification changes that may result between DCT scans, qualitatively this results in overlapping exterior sample boundaries.
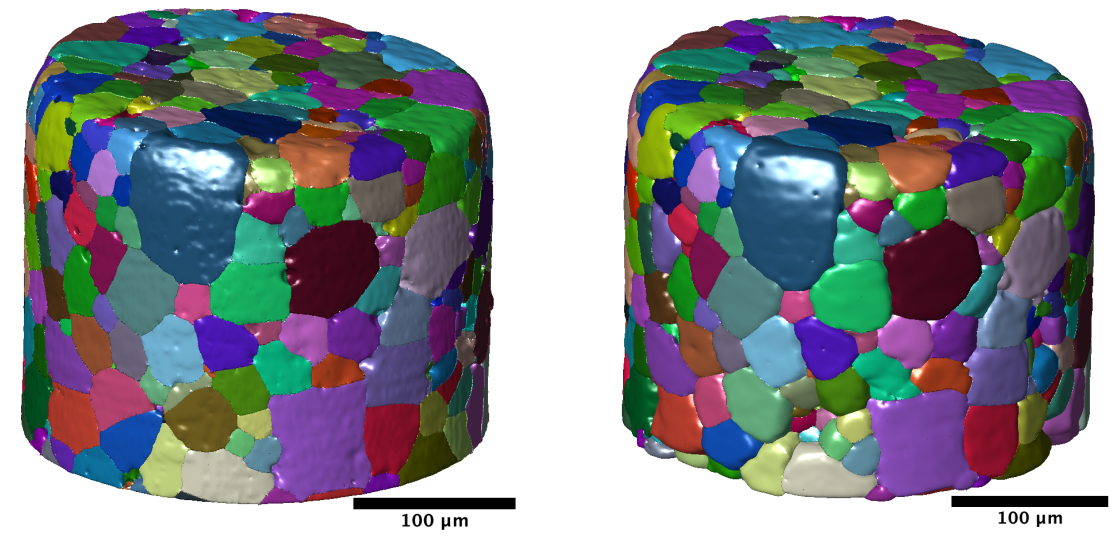

Fig. 1. 3D microstructure reconstruction of the investigated specimen (a) before and (b) after $1 \mathrm{~h}$ ex-situ annealing at $1600^{\circ} \mathrm{C}$. Grains are colored according to crystallographic orientation in a common reference frame.

All subsequent investigations are based on this subvolume, which contains a total of 849 grains in the initial state and 803 grains in the annealed state. A total of 404 grains can be considered as bulk grains (not impinging on the sample surface). All of them are found in both states. The 46 grains not found in the annealed state, are either located on the cylindrical surface or bottom/top of the specimen. All further analysis is based on the 404 bulk grains.

Comparing only the 404 bulk grains to the average relative volume change can be described as 


$$
\left\langle\frac{V-V_{0}}{V_{0}}\right\rangle_{b u l k}=\left\langle\frac{\Delta V}{V_{0}}\right\rangle_{b u l k}=-0.1
$$

where $V$ and $V_{0}$ are the volumes in the annealed and initial state respectively. The negative value corresponds to an overall shrinkage in total volume of the bulk grains. Correspondingly, the average grain size of the bulk grains shows a decrease from an average equivalent grain radius of $\bar{r}_{0}=14.4 \mu \mathrm{m}$ before annealing to $\bar{r}_{\text {anneal }}=14.0 \mu \mathrm{m}$ after annealing.

Before analyzing this structure any further it is worth discussing why a grain growth experiment leads to an average shrinkage of the grains analyzed here. To rationalize this behavior one has to recall some of the intricacies of the method. Due to the limited resolution of the DCT method, we do not find all the grains in the structure. Indeed, a higher resolution 3D-EBSD analysis finds more grains than the DCT reconstruction (Lenthe et al., 2015). Nevertheless the DCT reconstruction algorithm fills the entire volume with a dilatation step as described above. In the annealed state all the same grains are identified again and also dilated to fill the volume. If the total volume of these grains stayed the same we would consequently expect no average grain growth since all the shrinking grains are still identified. But we have only analyzed the interior bulk grains in the specimen and not taken the surface grains into consideration because the surface grains are expected to show different growth characteristics. Any grain that touches the surface is thereby considered a surface grain. A random $2 \mathrm{D}$-cut through a 3D grain structure inevitably sections through more large grains than small ones. Consequently our surface grains have an over-representation of large grains. These large grains then preferentially grow during the annealing step. Consequently, the layer of surface grains expands into the specimen and the total volume of the bulk grains is reduced. Therefore, the statistical information about the bulk grains must be looked at with some care, particularly with respect to mean values, but the individual 
grain behavior is expected to be well observed.
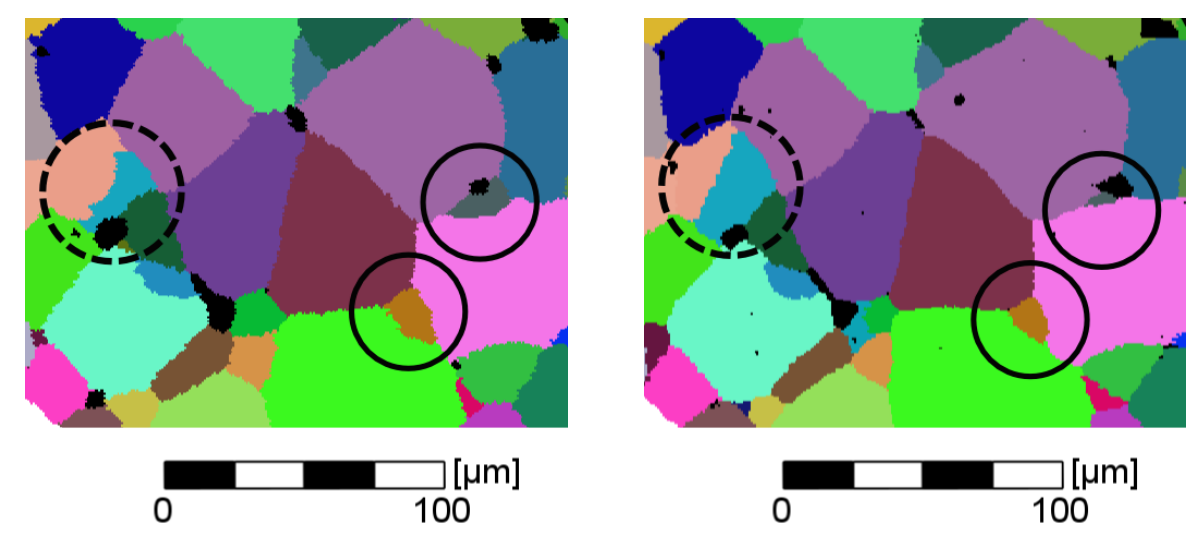

Fig. 2. Cross sections at same height of the microstructure reconstructions of (a) initial and (b) annealed state. Shrinking and growing grains are encircled with continuous and dashed lines, respectively.

Individual growing and shrinking grains in the evolving microstructure are highlighted in detailed views of cross-sections taken at the same height of the reconstructed volumes before and after annealing in Figures 2(a) and (b). The black areas in Figure 2 correspond to pores.

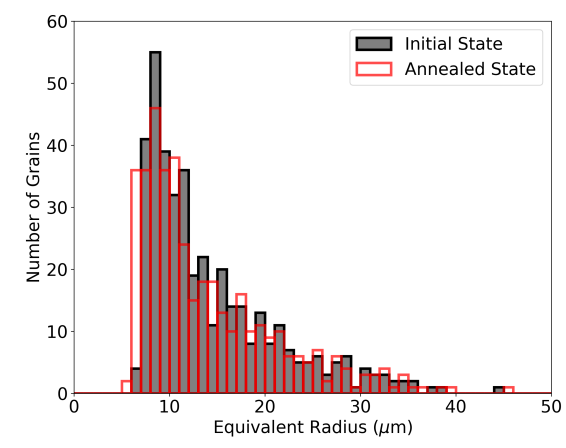

Fig. 3. Size distributions of bulk grains in the initial and annealed state of the specimen.

The grain size distributions are given in Figure 3. As expected, in the annealed state one finds some large grains that have apparently grown and some smaller grains at the IUCr macros version 2.1.6: 2014/01/16 
lower end of the resolved distribution. Absorption contrast tomography measurements showed a residual porosity of 1.4 vol- $\%$ for the specimen in the initial state. After annealing, phase contrast tomography, which detects more smaller pores due to higher resolution, revealed a volume fraction of 0.8 vol-\% porosity. (Values are recomputed and corrected versus previously reported data (Syha et al., 2012).) The pore volume has therefore significantly shrunk in this late stage of sintering and grain growth.

\subsection{Grain Topology}
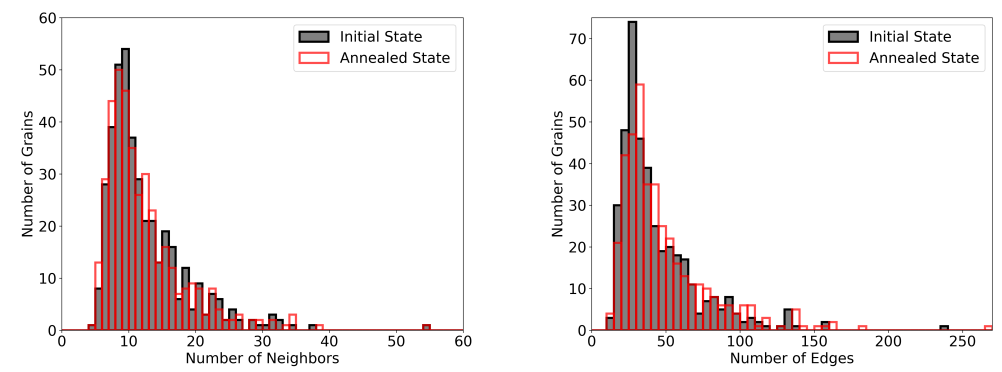

Fig. 4. (a) Distributions of number of neighbors per grain $F_{G}$ for both annealing states as bar diagrams. (b) Distributions of number of edges per grain $E_{G}$ for both annealing states as bar diagrams.

Figure 4(a) shows the distribution of the number of neighbors (number of interfaces) per grain $F_{G}$ for the bulk grains in both annealing states. The average number of neighbors per grain $\bar{F}_{G}$ remains almost constant, decreasing only marginally from $\left(\bar{F}_{G}=12.0\right)$ in the initial to $\left(\bar{F}_{G}=11.8\right)$ in the annealed state. Distributions of the number of edges per grain $E_{G}$ for both annealing states are given in Figure 4(b). The average number of edges per grain increases marginally from 31.1 to 32.2 in the annealed state. Both distributions do not seem to significantly change during annealing. 


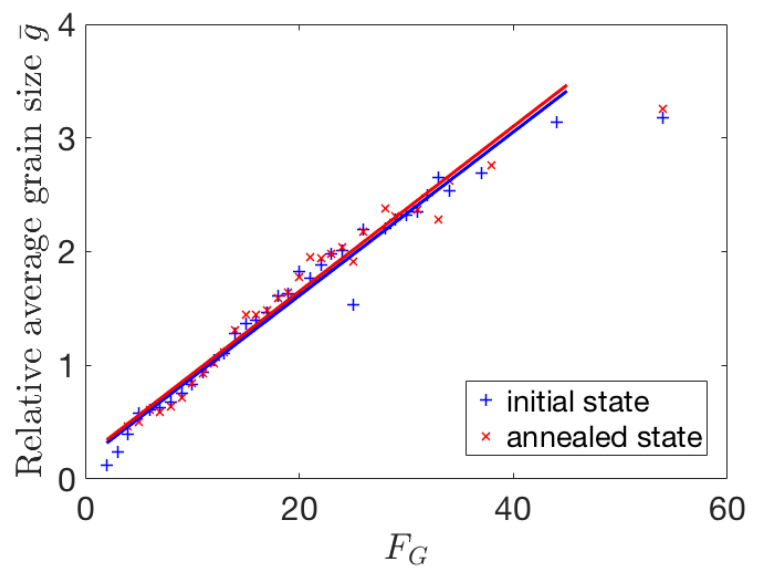

Fig. 5. Relative average grain radius $g_{C}$ plotted versus number of neighbors $F_{G}$ for the bulk grains in microstructure reconstructions of the initial and annealed state. Regression lines are given as continuous lines.

Figure 5 shows the relative average grain size $g_{C}=r / \bar{r}$ as a function of the number of neighbors $F_{G}$ for bulk grains contained in microstructure reconstructions of both annealing states. The relative grain size is averaged for a fixed number of neighbors per grain $F_{G}$. Disregarding the single, extraordinarily large grain with 54 neighbors, a linear correlation between $g_{C}$ and $F_{G}$ is obtained for both annealing states.

\subsection{Grain Morphology and Morphological Changes}

It was already found in (Syha et al., 2012) that many grains appear to have a rather cubic shape. Here we attempt to quantify this finding using moment invariants of the shapes. Moment invariants are combinations of moments that are unaffected by certain types of transformations (MacSleyne et al., 2008). The Cartesian central moments of an object in 3D are defined as:

$$
\mu_{p q r}=\iiint_{D} \mathrm{~d} x \mathrm{~d} y \mathrm{~d} z\left(x-x_{c}\right)^{p}\left(y-y_{c}\right)^{q}\left(z-z_{c}\right)^{r},
$$

where $x_{c}, y_{c}, z_{c}$ are its center-of-mass coordinates and the order of the moment $n$ is equal to the sum of the exponents $p+q+r . D(x, y, z)$ describes its shape or indicator 
function, such that $D=1$ inside the object and $D=0$ outside of it.

The independent 3D second order moment invariants (MIs) are (MacSleyne et al., 2008):

$$
\begin{aligned}
& \Omega_{1}=\frac{3 V^{5 / 3}}{\mu_{200}+\mu_{020}+\mu_{002}}, \\
& \Omega_{2}=\frac{3 V^{10 / 3}}{\mu_{200} \mu_{020}+\mu_{200} \mu_{002}+\mu_{020} \mu_{002}-\mu_{110}^{2}-\mu_{101}^{2}-\mu_{011}^{2}}, \\
& \Omega_{3}=\frac{V^{5}}{\mu_{200} \mu_{020} \mu_{002}+2 \mu_{110} \mu_{101} \mu_{011}-\mu_{200} \mu_{011}^{2}-\mu_{020} \mu_{101}^{2}-\mu_{002} \mu_{110}^{2}} .
\end{aligned}
$$

The first two moment invariants, $\Omega_{1}$ and $\Omega_{2}$, are invariant to similarity transformations, which include translation, rotation, and isotropic scaling. $\Omega_{3}$ is invariant to affine transformations like skewing and anisotropic scaling. For example, a sphere and an ellipsoid have $\Omega_{3}=1$ because an ellipsoid is simply an anisotropically scaled sphere.

The dimensionless moment invariants $\bar{\Omega}_{i}$ are normalized so that their values are between 0 and 1 , by dividing the dimensionless moment invariants by the expressions for the sphere. The moment invariants for the sphere are (MacSleyne et al., 2008):

$$
\Omega_{1}^{S}=\left(\frac{2000 \pi^{2}}{9}\right)^{1 / 3}, \Omega_{2}^{S}=\left(\frac{2000 \pi^{2}}{9}\right)^{2 / 3}, \Omega_{3}^{S}=\frac{2000 \pi^{2}}{9} .
$$

Some moment invariants for a few basic shapes are given in Table 1.

Table 1. Shape Descriptors for a few isotropic shapes (MacSleyne et al., 2008).

\begin{tabular}{c|ccc} 
& $\bar{\Omega}_{1}$ & $\bar{\Omega}_{2}$ & $\bar{\Omega}_{3}$ \\
\hline Sphere & 1 & 1 & 1 \\
Cube & 0.9236 & 0.8530 & 0.7879 \\
Tetrahedron & 0.7400 & 0.5477 & 0.4053 \\
Octahedron & 0.9324 & 0.8693 & 0.8106 \\
Dodecahedron & 0.9851 & 0.9705 & 0.9561 \\
Icosahedron & 0.9891 & 0.9782 & 0.9675
\end{tabular}

However, the authors should note that the magnitude of the Euclidean distance of $\Omega$ space is an challenging metric to interpret, where the degree of change of $\mathrm{D}$ may or may not be indicative of the degree of shape change, but that no change and or the direction of change is telling.

IUCr macros version 2.1.6: 2014/01/16 
To measure the distance D of a grain to a cube shape, one can first calculate the Euclidean distance of every individual grain from the moment invariants for a cube $\operatorname{are}\left(\bar{\Omega}_{1}^{c}, \bar{\Omega}_{2}^{c}, \bar{\Omega}_{3}^{c}\right)=(0.924,0.854,0.788)$ according to:

$$
D=\sqrt{\left(\bar{\Omega}_{1}^{g}-\bar{\Omega}_{1}^{c}\right)^{2}+\left(\bar{\Omega}_{2}^{g}-\bar{\Omega}_{2}^{c}\right)^{2}+\left(\bar{\Omega}_{3}^{g}-\bar{\Omega}_{3}^{c}\right)^{2}},
$$

where ()$^{g}$ refers to the value for a specific grain and ()$^{c}$ refers to the value for the cube. For reference, the distance between a cube and a sphere is 0.269 and can be considered as a fairly large distance between two shapes. It is found that the initial and annealed grains on average have about the same distance from a cube, with values of $0.137 \pm 0.077$ and $0.136 \pm 0.11$. So we do not find a general convergence towards cube shapes, which topologically would also be difficult to realize.
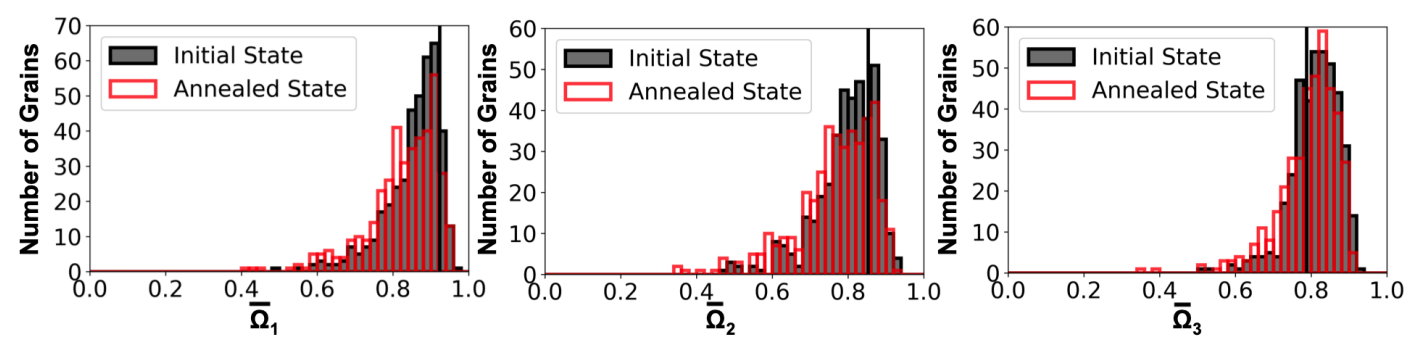

Fig. 6. Distribution of $\bar{\Omega}_{1}, \bar{\Omega}_{2}$, and $\bar{\Omega}_{3}$ before and after annealing. The average of each distribution is observed to shift from 0.853 to 0.825 for $\bar{\Omega}_{1}, 0.797$ to 0.767 for $\bar{\Omega}_{2}$ and 0.811 to 0.793 for $\bar{\Omega}_{3}$. The vertical lines indicate the values of $\bar{\Omega}_{1}, \bar{\Omega}_{2}$, and $\bar{\Omega}_{3}$ for a cube $(0.9236,0.8530$, and 0.788).

However, if one only focuses on $\bar{\Omega}_{3}$ as a shape descriptor which is invariant to affine transformations, one discriminates between the more rounded shapes (sphere, icosahedron, dodecahedron) with $\bar{\Omega}_{3}$ close to one, and the shapes with more pointed corners (octahedron, cube, tetrahedron) with values of 0.8 and below, one can observe a morphological evolution. Figure 6 displays the $\bar{\Omega}_{3}$ values of all grains before and after the annealing. Obviously, the annealing step has transformed some of the rounded IUCr macros version 2.1.6: 2014/01/16 
grains with large $\bar{\Omega}_{3}$-values closer towards $\bar{\Omega}_{3}=0.8$, the value of an octahedron or the cube.

If near cubic grains are dominated by low energy $\{100\}$ interfaces and these faces are also characterized by low mobility, as expected from the data in (Rheinheimer et al., 2015b), one expects these grains not to grow much after reaching a cubic shape. Of course, this can not be proven against other hypotheses but using the individual grain volume information, one can now probe this by calculating the relative volume change $\Delta V / V_{0}$ for each individual grain during the anneal step.

Figure 7 shows the relative volume change for all bulk grains during annealing as a function of the number of neighbors $F_{G}$. In addition, the 5 largest cube shaped grains (including grain \#100 in the upper left corner) are depicted as triangulated polyhedra (drawing not to scale) pointing to their corresponding relative volume change. It is apparent, that the relative volume change is small for all of these grains. The largest cube shaped grains were manually selected from large grains if they appeared qualitatively cubic upon visual inspection.

IUCr macros version 2.1.6: 2014/01/16 


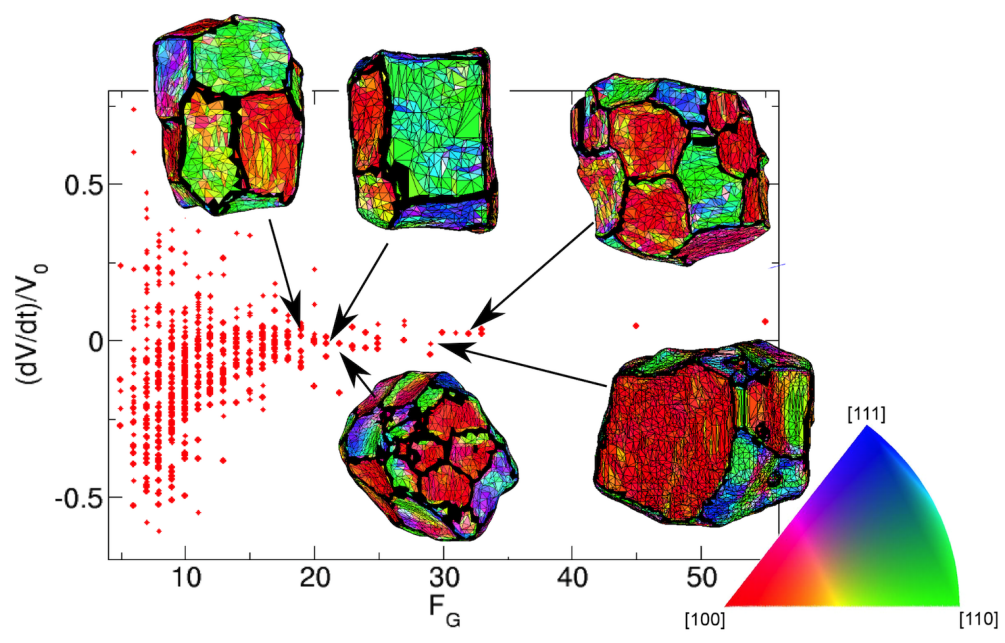

Fig. 7. Relative volume change $\Delta V / V_{0}$ as a function of the number of neighbors $F_{G}$. The 5 big grains with extraordinary flat interfaces are also shown with arrows pointing to their corresponding $\Delta V / V_{0}$ value. The grains are visualized as triangulated polyhedra. Black triangles indicate triple lines. Coloring is according to the meshed grain boundary normal orientation, as shown in the legend.

\subsection{Orientation of grains and interfaces}

The grain boundary plane distribution of bulk grains is calculated from the voxel information of the reconstructed samples. To obtain a smooth representation of the grain boundaries, they are triangulated and smoothed using MATLAB isosurface (The MathWorks, 2014) as shown in Figure 7. The grain boundary interface orientation is then calculated with respect to the crystallographic orientations of the two adjacent grains. Therefore two orientations are obtained for each triangle of one interface. In order to investigate how frequently specific local grain boundary interface orientations occur, the orientation space is discretized into bins of approximately $0.5^{\circ}$ in both azimuthal and polar angle coordinates. Using the cubic symmetry of the crystal structure, all grain boundary orientations are mapped onto the standard stereographic triangle. The observed distribution is normalized by the random orientation distribution function. The corresponding grain boundary plane distributions given in Figure 8 show a 
preference for grain boundaries with normal orientations within $15^{\circ}$ of a $<100>$ orientation. This preference is reflected in an excess of $\approx 15 \%$ with respect to the random distribution in the initial state and increases during annealing. In the annealed state the peak in the orientation distributions lies within $10^{\circ}$ of a $<100>$ orientation. Such orientations were found to be $\approx 20 \%$ more likely than in the random distribution.
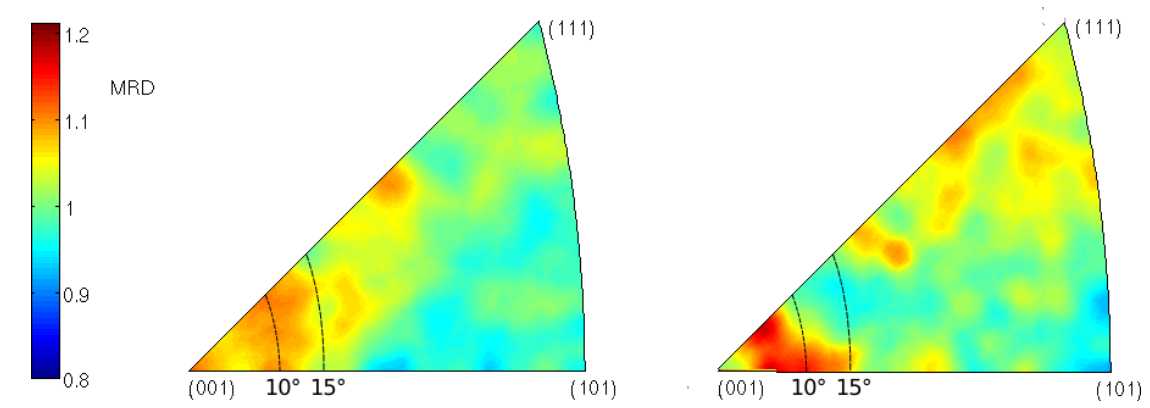

Fig. 8. Local grain boundary plane distributions for all bulk grains in the initial (left) and annealed (right) state given in multiples of the random distribution (MRD).

The preference for certain grain boundary plane orientations is also visible from $2 \mathrm{D}$ cross sections through the reconstruction. Figures 9 (a) and (b) show cross sections parallel to a (100)-plane of grain \#100 and some of its neighbors before and after annealing. The crystallographic orientations of the neighboring grains are indicated by tripods. The cutting plane is located at the median [100] coordinate. Red and green lines give the shape of grain \#100 at $10 \%$ of the grain diameter above and below the cutting plane. Thus overlapping lines indicate a grain boundary plane perpendicular to the cutting plane. From this information it becomes clear that nearly all cut faces of the grain are oriented parallel to the [100]-axes.

Special emphasis shall be drawn to the grain boundaries shared by grain \#100 and grain \#27 and the shared boundary between grain \#100 and grain \#170. These boundaries are almost parallel to the [010]- and [001]-axes, respectively, indicating a $<100>$ grain boundary normal orientation. Both boundaries appear to be significantly IUCr macros version 2.1.6: 2014/01/16 
closer to a straight $<100>$ orientation in Figure 9(b) illustrating the post-annealing state.
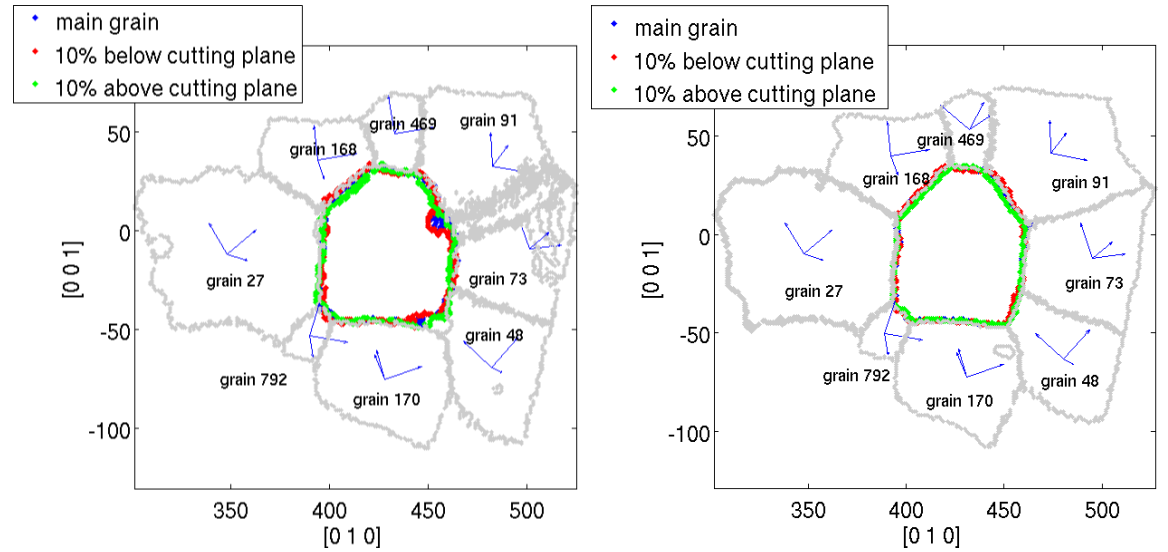

Fig. 9. 2D cut along the median (100)-plane of grain \#100 and neighboring grains (a) initially and (b) post-annealing. Green and red lines indicate the outline of the center grain when cut $10 \%$ of the grain diameter above and below the shown cross section.

The grain boundary misorientation angle distribution for the microstructure reconstruction in the initial state is found to be very close to the distribution of randomly textured polycrystals (Mackenzie, 1958) and does not change during annealing (shown in previous work (Syha et al., 2012)). Comparison of the misorientations of all 3850 internal interfaces in the initial and annealed state reveals a misorientation difference below $0.5^{\circ}$.

\subsection{Growth of individual grains}

The individual volume change of all bulk grains is displayed again in Figure 10. Figure 10 (a) shows the relative volume change $\Delta V / V_{0}$ as a function of the equivalent radius of the grain in the initial structure, Figure 10 (b) shows the total volume change as a function of the number of neighbors in the initial structure. Size and color of the spheres are chosen to indicate the maximum volume change of their neighbors. This helps us to identify neighborhood for a few grains.

IUCr macros version 2.1.6: 2014/01/16 
Isotropic 3D grain growth modelling (MacPherson \& Srolovitz, 2007) can give a rough estimate that on average grains with more than 15 neighbors(Rowenhorst et al., 2010; Zhong et al., 2017) are expected to grow, while smaller ones are expected to shrink. Clearly this problem is more complicated and that grain boundary curvature could also be incorporated, however, for simplicity we display the information in Figure 10. Obviously, our data in Figure 10 (b) shows many large grains with more than 15 neighbors that actually shrink and also many smaller grains with fewer than 15 neighbors that grow.
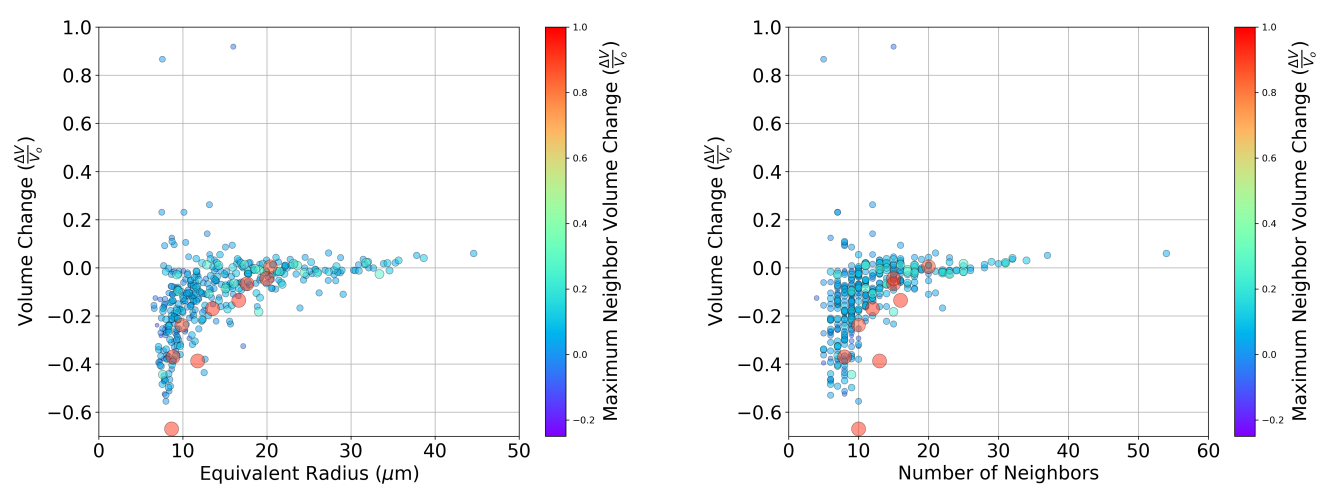

Fig. 10. Relative volume change plotted (a) versus radius and (b) versus number of neighbors. Size and color of markers according to the maximum volume change of their neighbors. (The grains in red are neighbors to one of the two grains which grew very significantly (\#801 and \#342). Only 9 grains are shown like this since both growing grains have several neighbors that themselves touch the surface and are therefore not included in the analysis.) 

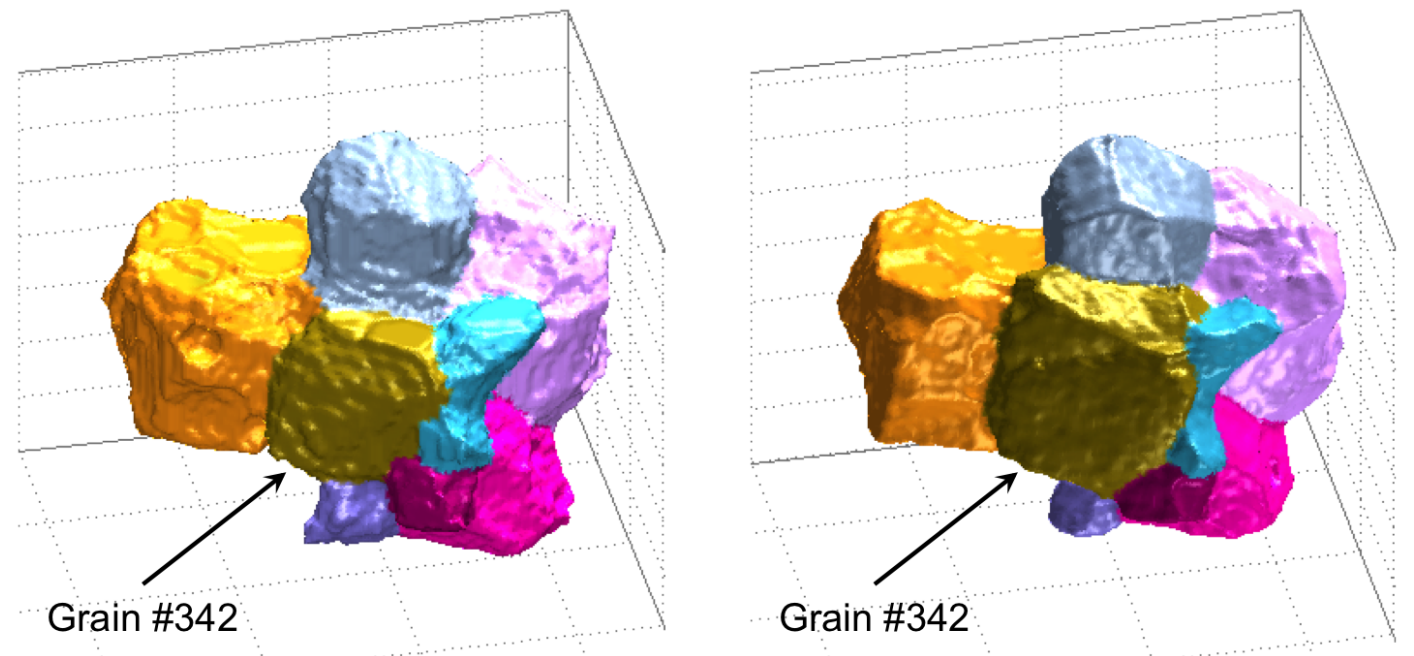

Fig. 11. Grain \#342 displayed in golden color and six neighboring grains (a) initially and (b) post-annealing. The coloring of the grains is only used to distinguish different grains.

Before analyzing individual grains in more detail it is worth noting that the largest five grains all grow and particularly \#1 grows by more than $6 \%$. Together they amass most of the overall volume gain of all grains. Then there are however two smaller grains which have almost doubled their size. For the smaller of them \#801, the actual volume growth is within the two voxel error margin in the determination of total volume. It is therefore not analyzed any further. However, grain \#342, a grain of just average size and with initially 15 neighbors also grows very significantly. It collects the second largest absolute volume gain after grain \#1.

Grain \#342 and some of its neighbors are displayed in Figure 11. Because of the unusual behavior of grain \#342 it was very carefully inspected to ensure that no reconstruction artifacts or particularly large dilatation or missing neighboring grains have been misinterpreted as significant growth. None of this has occurred. Grain \#342 remains consistently connected to all its 15 neighbors and gains four new neighbors during the growth process. It gains volume from all but three of these neighbors. The IUCr macros version 2.1.6: 2014/01/16 
only apparent specifics that grain \#342 shows are that some of its grain boundaries in the initial structure have an orientation close to a $\{111\}$ plane, which is unexpected given the average population of $\{111\}$ interfaces in the entire sample. It does not have any faces that are close to $\{100\}$ facets. In the annealed state the pronounced $\{111\}$ over-representation has almost disappeared, but $\{100\}$ faces are still significantly underrepresented.

\section{Discussion}

The results presented above demonstrate the feasibility of a time dependent 3D microstructure characterization of a polycrystalline ceramic material using X-ray DCT. The individual grains could be re-identified after heating so that the two microstructure reconstructions could be aligned following the procedures outlined in (Lenthe et al., 2015). This allows for a grain-by-grain investigation of volume, orientation and topology characteristics of several annealing states. For each individual grain, changes in all these characteristic quantities are determined. In combination with $3 \mathrm{D}$ grain growth simulations a long term goal is to aim at the validation of grain growth models. However, before doing so we first have to gain confidence in the DCT reconstruction and in its subsequent application to the same specimen, the central aim of this paper.

The porosity data for strontium titanate reported in the literature for similar heat treatments are below 1\% (Bäurer et al., 2009b), which is in good agreement with the $0.8 \% \mathrm{PCT}$ result in the annealed state in the current investigation. The difference in the initially higher porosity of $1.4 \%$ may be caused by segmentation accuracy, which itself is influenced by noise characteristics of the raw data (Dzieciol et al., 2013). Generally, a higher contrast leads to more realistic pore shapes. Thus, the 0.8 vol- $\%$ porosity obtained by phase contrast tomography measurements in the annealed state is likely to be more precise than the value of 1.4 vol- $\%$ obtained from absorption

IUCr macros version 2.1.6: 2014/01/16 
tomography in the initial state.

Tomography specimens must have a relatively small volume for X-ray transparency as well as for the ability to reconstruct the entire $3 \mathrm{D}$ volume. We have used a specimen with a diameter of about $300 \mu \mathrm{m}$ and an average grain diameter of about $30 \mu \mathrm{m}$ in order to get about 1000 initial grains with sufficient resolution. These parameters were chosen to get into a safe regime, where we would get good DCT data and be able to reconstruct and model the $3 \mathrm{D}$ dataset.

Unfortunately, this resulted in a large fraction of grains, about half of them, intersecting the specimen surface. Surface grooving pins grain boundaries at their location on the surface. The consequences of this pinning might actually reach deeper than the one layer of surface grains that has been excluded from the analysis here (Zhong et al., 2019). To exclude more grains deeper below the surface, while not statistically biased towards larger grains (see section 3.1), would require the exclusion of a layer of thickness at least the diameter of the largest grains in the specimen. Our specimen was simply too small to do this. Consequently, our dataset can not be used to extract statistically significant grain growth parameters.

We conclude here that despite the advent of non-destructive 3D techniques like DCT, a statistically meaningful measurement of grain growth remains inherently difficult on small samples to which 3D reconstruction can be applied. Even on large bulk specimens, amenable only to $2 \mathrm{D}$ analysis, statistical errors on the order of a factor of two are often reported (Bäurer et al., 2009b). Other approaches to capture grain growth include studies that look at curvature (Rowenhorst et al., 2006) via morphological phase evolution, but do not capture crystallographic effects, and those that carefully investigate grain boundary character distributions (Zhong et al., 2019; Zhong et al., 2017), but do not have time evolution. Ideally crystallographic, boundary curvature, and grain boundary character distributions all will be captured for detailed

IUCr macros version 2.1.6: 2014/01/16 
grain growth studies.

The investigation of the topology of the bulk material and its evolution in $3 \mathrm{D}$ yields an average number of neighbors $\bar{F}_{G}$ of approximately 12 in both annealing states. The average number of edges per grain $\bar{E}_{G}$ was found to be 31.1 in the initial and 32.2 in the annealed state. A comparison of these quantities with the values obtained from vertex dynamics simulations $\left(\bar{F}_{G}=13.6 \pm 0.2, \bar{E}_{G}=35.1 \pm\right.$ 0.1) (Syha, 2014) and statistical grain models such as Coxeter polytopes (Coxeter, 1987) $\left(\bar{F}_{G}=13.7, \bar{E}_{G}=34.9\right)$, Kelvin's $\alpha$-tetrakaidecahedra (Smith, 1964) or Williams' $\beta$-tetrakaidecahedra (Williams, 1968) (both $\bar{F}_{G}=14.0, \bar{E}_{G}=35.7$ ) shows that the average number of neighbors and the average number of edges per grain in our 3D reconstruction appear to be somewhat lower than the values obtained in the simulations. This could be due to fact that large grains have a higher probability of intersecting the sample surface and thus are excluded from the analysis. It could also be due to a certain amount of anisotropy in our grain shapes, or the existence of several flat pores between two grains. Although we removed these pores for neighbor counting, they may still have influenced the 3D microstructure.

A linear relation between relative average grain size $g_{C}$ and the number of neighbors $F_{G}$ is found for the reconstructed microstructure in both annealing states, see Figure 5. This is in good agreement with findings in other sintered materials (German, 2010; Tewari et al., 1999). Structures with predominantly spherical grains, where the number of faces is proportional to the grain surface area, show a declining rise in relative average grain size $g_{C}$ with increasing number of faces $F_{G}$ (Rowenhorst et al., 2010). This suggests, that the overall grain shape deviates from a sphere, which is supported by the moment invariant analysis. Indeed, a slight evolution towards less spherical grains is observed here during annealing as shown in Figure 6.

In the grain size distribution Figure 3, we observe a slight shift towards smaller

IUCr macros version 2.1.6: 2014/01/16 
grain sizes in the annealed state. This is due to the higher imaging resolution used to measure the annealed state sample, revealing more of the bulk grains from the initial structure, despite the fact that some of them have considerably shrunk. With the 3D EBSD method (Lenthe et al., 2015) yet more small grains are found in the annealed structure.

However, the overall appearance of the grain growth data in the investigated specimen points to some unusual grain growth behavior. The unusual grains deviate from the typical population morphology. The size change of the individual bulk grains (Figure 10) clearly shows that most of the volumetric growth is contributed by very few grains, including a few comparably small ones, while the volume loss is contributed from many grains. In particular, the volume loss is contributed from many comparably large grains with more than 15 neighbors, which on average, according to isotropic 3D grain growth modelling (Syha \& Weygand, 2010; MacPherson \& Srolovitz, 2007; Rowenhorst et al., 2010) should not shrink but instead grow.

Abnormal grain growth in strontium titanate is often connected to the growth of grains with particularly straight $\{100\}$ grain boundary planes (Shih et al., 2010), or even larger interfaces of single-crystalline seeds growing into a polycrystalline matrix (Rheinheimer et al., 2015b). Transmission electron microscopy observations indicate that the grain boundaries are curved close to triple lines. Boundary segments away from these triple lines can be atomically flat over micrometers (Bäurer et al., 2010; Shih et al., 2010). In the present study, no such well developed abnormal grains with flat boundaries were present. But already the initial state reveals grains with a global outer shape close to a $<100>$ oriented cube, see Figure 7 . While the global interface orientations in these cube-like grains are perpendicular to $<100>$ directions with respect to the grain reference frame, these faces are not completely flat and are composed of several grain boundaries separated by triple lines. Similar observations have

IUCr macros version 2.1.6: 2014/01/16 
been made for grain boundaries surrounding abnormally grown grains in strontium titanate (Shih et al., 2010). There, the general interface orientation is perpendicular to $<100>$ orientations of the abnormally grown grains. Of course, the post mortem analysis in (Shih et al., 2010) can not determine the face that actually grew. In the structure investigated here, this is different. We find that large grains indeed have pronounced flat, near $\{100\}$ faces but we find rather low relative growth rates for these big grains with $\{100\}$ interfaces (see Figure 7). We also do not find individual $\{100\}$ faces to contribute significantly to the growth of fast-growing grains (e.g. \#342, Figure 11). This rather points to a reduced probability for growth on flat $\{100\}$ surfaces which is also directly observed on the selected grains shown in Figure 9, which is in perfect agreement with the grain growth studies in (Rheinheimer et al., 2015a) which gave a distinctly low growth velocity for $\{100\}$ interfaces.

A preference for $\{100\}$ grain boundary planes was found in microstructure reconstructions of both annealing states, see Figure 8. These planes were identified as minimum energy planes both in first-principle and mean field energy calculations (Padilla \& Vanderbilt, 1998; Pojani et al., 1999) as well as in geometric and crystallographic measurements of grain boundary thermal grooves (Sano et al., 2003) and inverse Wulff constructions based on the investigations of intragranular pores in the same material (Rheinheimer, 2013; Rheinheimer et al., 2015a; Rheinheimer et al., 2017). Similar grain boundary plane distributions were obtained by SEM and EBSD measurements in strontium titanate at $1425^{\circ} \mathrm{C}$ (Rheinheimer et al., 2015 a), $1470^{\circ} \mathrm{C}$ (Miller \& Rohrer, 2008) and $1650^{\circ} \mathrm{C}$ (Saylor et al., 2004b; Saylor et al., 2004a). In all of these studies, the $\{100\}$ low energy interfaces were found to dominate the distribution of grain boundary planes, which is in excellent agreement with the results presented in this paper. However, none of these works reported a change in the grain boundary plane distribution with annealing time that we find (Figure 8). This might be due to a

IUCr macros version 2.1.6: 2014/01/16 
much larger bin size in these studies $\left(10^{\circ}\right.$ as compared to $0.5^{\circ}$ in the present investigation), so that a slight displacement of the orientation peak, as it was observed in the present study, could not be resolved. It seems worth mentioning here that our study does not give any distinct grain boundary orientation maxima at other than the $\{100\}$ orientation, while other surface energy minima, namely at $\{110\}$ and $\{111\}$ surfaces, are identified in the above mentioned studies.

Abnormal grain growth is classically measured from statistical grain growth analysis and should not occur at such high annealing temperatures (Bäurer et al., 2009b; Rheinheimer et al., 2019) as applied here. Obviously, the statistical analysis of our sample in Figures 3, 4 and 5 did not pick up any anomaly and only the grain-by-grain analysis of the growth behavior showed "unusual" behavior. The "unusualness" is seen mostly by the fact that several medium sized grains and particularly grain \#342 preferentially grew. Figure 10 shows that several of the shrinking grains are direct neighbors to this grain. A few of the yet larger shrinking grains are neighbors to grain \#1. In this sense the "abnormality" is seen rather in the growth of these grains than in the shrinkage of the other relatively large grains. It, of course, still remains unclear what gives the growth advantage to the few growing grains. It is clearly not the $\{100\}$ interface orientations since the individual grains that grew very significantly, grain \#1 and grain \#342, preferentially overgrew other grain boundary orientations and even in the annealed state had a deficiency of $\{100\}$ interface orientations.

We can not exclude that the growth behavior still is a consequence of the anisotropic grain boundary properties. The shape evolution towards low energy $\{100\}$ grain boundaries and the limited change in the volume of the "cubic" grains, which both clearly indicate anisotropic grain boundary properties, may have frustrated certain grain boundary configurations out of which a few other grains, precisely not of these orientations, could benefit. To clarify this situation, grain growth simulations should be 
run on exactly the observed structures to discriminate "normal" from "abnormal" behavior.

In summary, the feasibility of non-destructive time-dependent microstructure characterization, which is demonstrated in this study, opens the possibility to a grain-bygrain analysis of the behavior of individual grains in their given environment. This allows for systematic correlation of the behavior of the grains to their specific neighborhood and crystallographic orientation of their interfaces.

In the future these studies can and should be extended both in terms of the investigated volume (number of grains) as well as towards multiple time steps in the annealing process. They may also be used to further investigate the origin of the different grain growth behavior at different temperatures and possibly lead to new insights on the role of faceting of individual grain boundaries. However, due to the inherent difficulty in obtaining 3D tomographic information on large samples, and due to unavoidable surface effects, future studies will remain limited in their statistical significance. Nevertheless, they will be extremely useful if they can be directly compared to simulations of the evolution of exactly the observed microstructures. Multiple time step investigation will then of course be needed, but it may then become feasible to extract specific materials properties like grain boundary energy and mobility from the direct simulation of the measured evolution of such microstructures.

\section{Conclusions}

Diffraction contrast tomography has been demonstrated to allow the investigation of the time resolved evolution of the 3D grain structure of a strontium titanate specimen. The achieved spatial resolution yields the measurement of both volumetric and interfacial properties.

The grain boundary orientation distribution function shows an increase of grain IUCr macros version 2.1.6: 2014/01/16 
boundary orientations close to the $\{100\}$ plane of one of the two grains forming a grain boundary. This preferred interface orientation coincides with the low energy orientation of the surface energy of strontium titanate. Several grains, particularly the larger ones, became more cuboidal during annealing and their near $\{100\}$ grain boundaries are flattening and straightening towards more ideal $\{100\}$ orientation. These grains, however, showed low average growth rates.

The microstructure evolution was dominated by the "abnormal" growth of a few grains and the corresponding shrinkage of many grains including several large grains.

Subsequent investigations must include multiple-timestep 3D characterizations of microstructure evolution and the direct comparison to grain growth simulations on the observed microstructures.

Acknowledgements This work was supported by the Deutsche Forschungsgemeinschaft (DFG) under grant number WE3544/4-1, GU367/30-1, and BA4143/2 and the European Synchrotron Radiation Facility (ESRF) by the provision of beam time. AFOSR / AFRL Grant Number FA9550-12-1-0445 is also acknowledged (WL, ME and TP).

\section{References}

Amaral, L., Fernandes, M., Reaney, I., Harmer, M., Senos, A. \& Vilarinho, P. (2013). Jorunal of Physical Chemistry, 117(47), 24787-24795.

Avrami, M. (1941). Journal of Chemical Physics, 9, 177-184.

Bäurer, M., Kungl, H. \& Hoffmann, M. (2009a). Journal of the American Ceramic Society, 92(3), 601-606.

Bäurer, M., Störmer, H., Gerthsen, D. \& Hoffmann, M. (2010). Advanced Engineering Materials, 12, 1230-1234.

Bäurer, M., Weygand, D., Gumbsch, P. \& Hoffmann, M. (2009b). Scripta Materialia, 61(6), $584-587$.

Burke, J. \& Turnbull, D. (1952). Progress in Metal Physics, 3, 220-291.

Chung, S. \& Kang, S. (2003). Acta Materialia, 51, 2345-2354.

Cloetens, P., Pateyron-Salomé, M., Buffière, J. Y., Peix, G., Baruchel, J., Peyrin, F. \& Schlenker, M. (1997). Journal of Applied Physics, 81, 5878.

Coxeter, H. (1987). Regular Polytopes. Macmillan Company.

Dzieciol, K., Borbely, A. \& Scheel, M. (2013). Journal of Physics: Conference Series, 425, 192005.

German, R. (2010). Critical Reviews in Solid State and Material Sciences, 35(4), 263-305.

IUCr macros version 2.1.6: 2014/01/16 
Groeber, M. \& Jackson, M. (2014). Integrating Materials and Manufacturing Innovation, 3(1), 5 . URL: http://www.immijournal.com/content/3/1/5

Gumbsch, P., Taeri-Baghbadrani, S., Brunner, D., Sigle, W. \& Rühle, M. (2001). Physical Review Letters, 87, 085505.

Hall, E. (1954). Nature, 173, 948-949.

Hillert, M. (1965). Acta Metallurgica, 13(3), 227-238.

Holm, E. \& Bataille, C. (2012). Journal of the Minerals, Metals and Materials Society, 53(9), 20-23.

Kang, S., Chung, S. \& Nowotny, J. (2003). Key Engineering Materials, 253, $63: 72$.

Lee, S., Sigle, W., Kurtz, W. \& Rühle, M. (2003). Acta Materialia, 51, 975-981.

Lenthe, W. C., Echlin, M. P., Trenkle, A., Syha, M., Gumbsch, P. \& Pollock, T. M. (2015). Journal of Applied Crystallography, 48(4), 1034-1046. URL: https://doi.org/10.1107/S1600576715009231

Ludwig, W., Reischig, P., King, A., Herbig, M., Lauridsen, E., Johnson, G., Marrow, T. \& Buffière, J. (2009). Review of Scientific Instruments, 80(3), 033905.

Ludwig, W., Schmidt, S., Lauridsen, E. \& Poulsen, H. (2008). Journal of Applied Crystallography, 41, 302-309.

Mackenzie, J. (1958). Biometrika, 45, 229-240.

MacPherson, R. \& Srolovitz, D. (2007). Nature, 446, 1053-1055.

MacSleyne, J. P., Simmons, J. P. \& Graef, M. D. (2008). Modelling and Simulation in Materials Science and Engineering, 16(4), 045008.

URL: $h$ ttp://stacks.iop.org/0965-0393/16/i=4/a=045008

Miller, H. \& Rohrer, G. (2008). Evolution of the Grain Boundary Character Distribution in Strontium Titanate during Grain Growth, pp. 335-342. New York: Wiley.

Padilla, J. \& Vanderbilt, D. (1998). Surface Science, 418, 64-70.

Pojani, A., Finocchi, F. \& Noguera, C. (1999). Surface Science, 442, 179-198.

Reischig, P., King, A., Nervo, L., Vigano, N., Guilhem, Y., Palenstijn, W., Batenburg, K., Preuss, M. \& Ludwig, W. (2013). Journal of Applied Crystallography, 46, 297-311.

Rheinheimer, W. (2013). Zur Grenzflächenanisotropie von $\mathrm{SrTiO}_{3}$. Universitätsverlag Karlsruhe.

Rheinheimer, W., Altermann, F. J. \& Hoffmann, M. J. (2017). Scripta Materialia, 127, 118 121.

URL: https://doi.org/10.1016/j.scriptamat.2016.09.017

Rheinheimer, W., Bäurer, M., Chien, H., Rohrer, G. S., Handwerker, C. A., Blendell, J. E. \& Hoffmann, M. J. (2015a). Acta Materialia, 82, 32-40.

URL: https://doi.org/10.1016/j.actamat.2014.08.065

Rheinheimer, W., Bäurer, M., Handwerker, C. A., Blendell, J. E. \& Hoffmann, M. J. (2015b). Acta Materialia, 95, 111-123.

URL: https://doi.org/10.1016/j.actamat.2015.05.019

Rheinheimer, W. \& Hoffmann, M. J. (2015). Scripta Materialia, 101, 68-71. URL: https://doi.org/10.1016/j.scriptamat.2015.01.021

Rheinheimer, W., Schoof, E., Selzer, M., Nestler, B. \& Hoffmann, M. J. (2019). Acta Materialia, 174, 105-115.

URL: https://doi.org/10.1016/j.actamat.2019.05.040

Rowenhorst, D., Kuang, J., Thornton, K. \& Voorhees, P. (2006). Acta Materialia, 54(8), $2027-2039$.

URL: https://doi.org/10.1016/j.actamat.2005.12.038

Rowenhorst, D., Lewis, A. \& Spanos, G. (2010). Acta Materialia, 58(16), 5511-5519.

URL: https://doi.org/10.1016/j.actamat.2010.06.030

Russ, J. C. \& Dehoff, R. T. (2000). Practical Stereology. Springer US.

URL: https://doi.org/10.1007/978-1-4615-1233-2

IUCr macros version 2.1.6: 2014/01/16 
Sano, T., Saylor, D. \& Rohrer, G. (2003). Journal of the American Ceramic Society, 86(11), 1933-1939.

Saylor, D., Dasher, B., Pang, Y., Miller, H., Wynblatt, P., Rollett, A. \& Rohrer, G. (2004a). Journal of the American Ceramic Society, 87(4), 724-726.

Saylor, D., Dasher, B., Sano, T. \& Rohrer, G. (2004b). Journal of the American Ceramic Society, 87(4), 670-676.

Shih, S., Lozano-Perez, S. \& Cockayne, D. (2010). Journal of Materials Research, 25(2), $260-265$.

Smith, C. (1964). Metallurgical Reviews, 9, 1-48.

Syha, M. (2014). Microstructure Evolution in Strontium Titanate-Investigated by means of Grain Growth Simulations and X-ray Diffraction Contrast Tomography Experiments. Universitätsverlag Karlsruhe.

Syha, M., Rheinheimer, W., Bäurer, M., Lauridsen, E., Ludwig, W., Weygand, D. \& Gumbsch, P. (2012). Scripta Materialia, 66(1), 1-4. URL: $h$ ttps://doi.org/10.1016/j.scriptamat.2011.08.005

Syha, M., Trenkle, A., Lödermann, B., Graff, A., Weygand, D., Ludwig, W. \& Gumbsch, P. (2013). Journal of Applied Crystallography, 46, 1145-1150.

Syha, M. \& Weygand, D. (2010). Modelling and Simulation in Materials Science and Engineering, 18(1), 015010.

Tewari, A., Gokhale, A. \& German, R. (1999). Acta Materialia, 47, 3721-3734.

The MathWorks, I., (2014). MATLAB, Isosurface Function.

Williams, R. (1968). Science, 161(3838), 276-277.

Zhang, J., Zhang, Y., Ludwig, W., Rowenhorst, D., Voorhees, P. W. \& Poulsen, H. F. (2018). Acta Materialia, 156, 76-85.

URL: https://doi.org/10.1016/j.actamat.2018.06.021

Zhong, X., Kelly, M. N., Miller, H. M., Dillon, S. J. \& Rohrer, G. S. (2019). Journal of the American Ceramic Society.

URL: https://doi.org/10.1111/jace.16608

Zhong, X., Rowenhorst, D. J., Beladi, H. \& Rohrer, G. S. (2017). Acta Materialia, 123, 136145.

URL: https://doi.org/10.1016/j.actamat.2016.10.030

IUCr macros version 2.1.6: 2014/01/16 